\title{
Involuntary autobiographical memories in and outside the laboratory: How different are they from voluntary autobiographical memories?
}

\author{
Simone Schlagman \\ Technical University of Dresden, Dresden, Germany \\ AND \\ Lia KVAVILASHVILI \\ University of Hertfordshire, Hatfield, England
}

\begin{abstract}
In two studies, we compared the characteristics and retrieval times of involuntary and voluntary autobiographical memory under controlled laboratory conditions. A new laboratory task of involuntary autobiographical memories involved detecting vertical lines in a stream of stimuli with horizontal lines (an undemanding vigilance task) and recording any involuntary memories during the session. The majority of these memories were reported as being triggered by irrelevant cue phrases presented on the screen. Voluntary autobiographical memories were sampled via a standard word-cue method in Session 2. The results showed that involuntary memories were more specific and were retrieved significantly faster than voluntary memories. They were also more likely to be triggered by negative cues, whereas cue valence did not have any effect on the number of voluntary memories. Furthermore, laboratory involuntary memories did not differ from naturalistic involuntary memories recorded in a diary by the same participants (Study 2). Taken together, these results have important implications for current theories of autobiographical memory, and they open up interesting avenues for future research.
\end{abstract}

Research on autobiographical memory is primarily based on a method in which participants deliberately recall personally experienced past events in response to a particular word or phrase provided by the experimenter (the so-called word-cue technique; see Conway \& Bekerian, 1987; Haque \& Conway, 2001; Rubin, 2005). Alternatively, participants may be asked to recall their most vivid or most important memories (Rubin \& Kozin, 1984; Rubin \& Schulkind, 1997b). However, autobiographical memories can also come to mind spontaneously without any conscious or deliberate attempt to retrieve them. Despite their prevalence in everyday life, these involuntary autobiographical memories have received relatively little attention, with only a dozen published empirical studies on the topic (e.g., Ball, 2007; Ball \& Little, 2006; Berntsen, 1996, 1998; Berntsen \& Hall, 2004; Berntsen \& Rubin, 2002; Hall \& Berntsen, 2008; Kvavilashvili \& Mandler, 2004; Mace, 2004, 2005, 2006; Schlagman, Kvavilashvili, \& Schulz, 2007; Watkins, Grimm, Whitney, \& Brown, 2005). Almost all of these studies have used the diary and questionnaire methods of enquiry.

Results from these initial diary studies indicate that there are certain optimal conditions in which involuntary memories occur in everyday life. First, they are more likely to come to mind while the person is engaged in fairly routine, automatic activities that require little attention - for exam- ple, making a cup of tea or brushing one's teeth (Berntsen, 1998; Kvavilashvili \& Mandler, 2004; Schlagman et al., 2007). second, the majority of memories are elicited by easily identifiable cues, most of which correspond to and form central features of the memory content (Berntsen, 1998; Berntsen \& Hall, 2004; Schlagman et al., 2007). Cues have also been found to be predominantly external (i.e., present in the environment), as opposed to internal thoughts (Ball \& Little, 2006; Berntsen, 1998; Berntsen \& Hall, 2004; Kvavilashvili \& Mandler, 2004; Mace, 2004; Schlagman et al., 2007). Furthermore, Berntsen (2007) reported that the majority of external cues from her 1998 diary study (i.e., 66\%) were peripheral aspects of the environment - that is, aspects of the environment that were not directly related to the current activity of the rememberer.

Another set of important findings concerns the characteristics of involuntary autobiographical memories. For example, involuntary memories have been repeatedly found to be of specific events as opposed to general events (Ball \& Little, 2006; Berntsen, 1998; Berntsen \& Hall, 2004; Schlagman et al., 2007). This predominance of specific events is in sharp contrast to mainstream research on voluntary autobiographical memories, whereby people often recall general events, even when explicitly asked to provide specific memories (Barsalou, 1988; Linton, 1986; Williams \& Dritschel, 1992).

L. Kvavilashvili, I.kvavilashvili@herts.ac.uk 
At present, there are only three studies that have directly compared involuntary and voluntary autobiographical memories within one sample of participants (Ball, 2007; Berntsen \& Hall, 2004; Mace, 2006). In all three studies, the percentage of reported specific involuntary memories was reliably higher than the percentage of specific voluntary autobiographical memories (see also Berntsen, 1998, who obtained a similar finding using a between-subjects design). However, findings concerning other memory characteristics have been less consistent. For example, Berntsen (1998) reported that involuntary memories were more positive and recent but less rehearsed than voluntary memories. These findings were not replicated in a study by Berntsen and Hall (2004); no differences were observed in age of memories or prior rehearsal, but involuntary memories were less positive and more unusual than voluntary memories.

It is obvious that more systematic research is needed to examine potential differences and similarities between these two forms of memory. According to Mace (2007), this "is perhaps the most challenging question facing involuntary memory research" (p. 7). Of particular interest is to compare the retrieval times of involuntary and voluntary memories, since this may provide important insights into the underlying mechanisms of involuntary memories, as well as into the structure and organization of the autobiographical memory system. However, in contrast with voluntary autobiographical memories, no information is currently available about the retrieval times of involuntary memories, because of the absence of a suitable laboratory paradigm. In order to achieve this aim, the studies presented in this article simulated the conditions of naturally occurring involuntary memories (i.e., diffused attention and easily identifiable cues), which allowed the capture of involuntary memories "online" and the measurement of their retrieval times.

In order to create a state of diffused attention in the laboratory, participants had to carry out an undemanding and monotonous vigilance task, which involved detecting infrequently presented vertical lines in a stream of stimuli with horizontal lines. Previous research by Giambra $(1989,1995)$ showed that undemanding vigilance tasks reliably induce task-unrelated thoughts or mind wandering, which are characteristic of many everyday habitual activities, such as washing up or brushing one's teeth (see also Singer, 1993; Smallwood \& Schooler, 2006). In addition to the vigilance task, however, we simultaneously presented written phrases, such as going on holiday, childhood nightmares, and buttering bread on the computer screen. The phrases were irrelevant to the ongoing vigilance task, since participants were instructed to ignore them (see the Method section), but it was expected that at least some of these phrases would elicit involuntary autobiographical memories. If an involuntary memory occurred during the task, then the participant indicated this by pressing the specified key, which stopped/ paused the task while he or she recorded the memory. The participant then returned to the vigilance task, repeating the procedure each time an involuntary memory was experienced.
The results of an initial pilot study using this procedure showed that participants did report experiencing involuntary memories during the vigilance task (Kvavilashvili \& Schlagman, 2003). Most importantly, the majority of elicited memories were reported to be triggered by cue phrases presented on the screen, which made it possible to measure the retrieval times of these memories (time taken from the presentation of the cue phrase and the reported memory). Therefore, in order to compare involuntary and voluntary memories, two studies were conducted in which participants completed two sessions, one week apart. In the involuntary memory session, participants carried out the vigilance task, and in the voluntary session, they deliberately recalled memories in response to word phrases presented on the screen (i.e., traditional word-cue experiment).

\section{STUDY 1}

The primary objective of Study 1 was to compare the retrieval times of involuntary and voluntary memories. In line with previous research on autobiographical memory, it was predicted that the retrieval of voluntary memories in response to word cues would be a slow and effortful process that would take, on average, up to $10 \mathrm{sec}$ (see, e.g., Rubin, 1998). These slow retrieval times are thought to reflect the hierarchical organization of autobiographical memory, which incorporates information at different levels of specificity from very abstract — such as lifetime periods (e.g., when I was a PhD student) - to specific sensoryperceptual details of particular events. Therefore, it takes time to access the bottom of the hierarchy and construct a particular memory from available fragments of eventspecific knowledge (Conway \& Pleydell-Pearce, 2000).

In contrast, the retrieval of involuntary memories is considered to be a fast and automatic process that is mediated by a so-called "direct retrieval" mechanism (see Conway \& Pleydell-Pearce, 2000). This assumption is made on the basis of the observation that during voluntary retrieval, in response to word-cues, participants can occasionally retrieve specific memories within $2 \mathrm{sec}$ (see, e.g., Haque \& Conway, 2001; see also Conway \& Bekerian, 1987). To account for such unusually fast retrieval times, Conway (2005) proposed that these memories are likely to be directly retrieved from a separate pool of very recent memories (probably from the last $24 \mathrm{~h}$ or less) that have not yet been fully consolidated into the hierarchical structure of autobiographical memory. However, currently, there is no information about the retrieval times of those involuntary memories that come to mind when one is not in the retrieval mode (i.e., deliberately trying to recall memories in response to cue words). In addition, it is unclear whether these memories are directly retrieved from a separate pool of highly active recent memories, as was suggested by Conway. Initial evidence suggests that this may not be the case. For example, in a diary study of Ball and Little (2006), only 3\% of recorded involuntary memories were from the last $24 \mathrm{~h}$, with the majority of memories $(66 \%)$ being 2 or more years old.

The second objective was to compare the two types of memories in terms of several important memory charac- 
teristics, such as specificity, age of memory, rehearsal, emotional valence, and so on. In line with previous studies, it was predicted that involuntary memories would be more specific than voluntary memories (see, e.g., Ball \& Little, 2006; Berntsen, 1998; Berntsen \& Hall, 2004; Mace, 2006). However, no predictions were made for other memory characteristics, given the inconsistent findings obtained in two previous studies that compared involuntary diary and voluntary word-cued memories (see Berntsen, 1998; Berntsen \& Hall, 2004).

The final objective of Study 1 was to examine how the emotional valence of cues affects the number and nature of involuntary and voluntary memories. Thus, equal numbers of positive, neutral, and negative cue phrases were presented to participants during both involuntary and voluntary memory sessions. A key question of interest was to see which cues are more likely to elicit involuntary memories. For voluntary memories, the predictions are relatively straightforward. Since the recall is deliberate and purposeful (i.e., retrieving a memory in response to a cue), participants should be equally likely to recall memories in response to positive, neutral, and negative cue phrases. This, however, may not be the case for involuntary memories in which recall is not attempted, and certain cues may be more likely to spontaneously elicit memories than others. For example, one may speculate that emotional cues may be better than neutral cues in eliciting involuntary memories. Alternatively, it can be predicted, on the basis of the evolutionary perspective, that negative cues are more important for survival and will thus be more likely to elicit involuntary memories than will positive and neutral cues (see, e.g., Baumeister, Bratslavsky, Finkenauer, \& Vohs, 2001). A related question was to examine the relationship between the valence of the cue phrase and the participants' own ratings of the emotional valence of their memories. If cues form part of the remembered event (as was shown by Berntsen [1998] and Schlagman et al. [2007]), then valence ratings should be highest for memories retrieved to positive cue words and lowest for memories retrieved to negative cue words, irrespective of memory type.

Method
Participants
Thirty-seven participants ( 14 males and 23 females) were re-
cruited from a pool of psychology undergraduates and by word of
mouth. Twenty-nine participants were students, and the remaining 8
had various professions, including lecturer, social worker, teacher,
and estate agent. Their ages ranged from 18 to 38 , with a mean age
of 22.73 ( $S D=5.63$ ). All of the participants spoke English as their
first language. They were paid $£ 5$ for each session, each session had
an average duration of approximately $1 \mathrm{~h}$. If the session lasted more
than 80 min, the participant was given an extra $£ 3$.

\section{Materials}

Session 1: Involuntary recall. The vigilance task was a 20-min program that was created with SuperLab software and presented on an Apple Macintosh laptop. The program consisted of 800 trials, each showing a card (approximately $21.5 \times 12.5 \mathrm{~cm}$ in size) depicting either a pattern of black horizontal or black vertical lines. The target stimuli appeared on 15 trials and consisted of a pattern of black vertical lines. The target patterns were randomly chosen from six basic patterns with between 4 and 8 vertical lines. The nontarget stimuli were presented on 785 trials and consisted of patterns of black horizontal lines. The nontarget patterns were randomly selected from 12 basic patterns, with between 4 and 8 lines. In addition to the pattern, a word phrase (e.g., relaxing on a beach; missed opportunity; crossing the road) was shown on each trial. The phrases were presented in 18-point Arial font and were placed in the middle of each card. They were used instead of single words (often used in voluntary autobiographical memory research), because they were more similar to types of cues reported by participants in our previous diary studies (e.g., My boyfriend mentioned Karvol drops or the newspaper article I was reading). The 800 trials were shown in a fixed order continuously, with each trial remaining on the screen for $1.5 \mathrm{sec}$. Target patterns occurred randomly every $60-90 \mathrm{sec}$ (i.e., 40-60 trials), in order to ensure that they came at fairly long and irregular intervals.

To control the emotional valence of cue phrases, a total of 1,492 phrases were generated by the authors and given to 8 independent coders to rate as negative, neutral, or positive. The percentage of agreement was taken as a measure of interrater reliability, and only phrases with an agreement of $75 \%$ or above were included, giving a pool of 1,176 cue phrases. Out of this pool, 800 phrases were randomly selected, and approximately equal numbers of negative $(n=$ $267)$, neutral $(n=266)$, and positive $(n=267)$ phrases were used.

Session 2: Voluntary recall. A total of 24 new phrases ( 8 negative, 8 neutral, and 8 positive) that had not been shown during Session 1 were randomly presented on an Apple Macintosh laptop with a program created by SuperLab software. Cue phrases were in 18-point Arial font and were shown in the middle of cards depicting black horizontal lines (taken from the nontarget stimuli with horizontal lines shown in Session 1). In addition to the 24 cue phrases, participants were also shown the cue phrases that had triggered their involuntary memories in Session 1 (the old and new phrases were intermixed and presented in random order). Therefore, the total number of cue phrases that were presented varied and was dependent on the number of memories reported during Session 1. The rationale was to examine whether the same memories would be voluntarily retrieved to the cues that had elicited involuntary memories earlier. However, in this article, only the data on 24 new cue phrases will be reported.

Imagery and concreteness of cue phrases. To ensure that cue phrases used in the voluntary and involuntary sessions were comparable, two new groups of participants rated the phrases for imagery $(n=14)$ and concreteness $(n=13)$ on a 7-point scale (ranging from 1 low to 7 high). The mean ratings as a function of cue type (involuntary, voluntary) and cue valence (negative, neutral, positive) were calculated for each participant and entered into two separate within-subjects ANOVAs, with concreteness and imagery as dependent variables. In both ANOVAs, there was no main effect of cue type $\left(F_{\mathrm{s}}<1\right)$ or a cue type $\times$ cue valence interaction $\left(F_{\mathrm{s}}<1\right)$. This result means that cue phrases used in voluntary and involuntary sessions were comparable in these characteristics. There was, however, a main effect of cue valence for both concreteness $[F(2,48)=31.87$, $\left.p<.001 ; \eta^{2}=.64\right]$ and imagery $\left[F(2,52)=43.94, p<.001 ; \eta^{2}=\right.$ .63]. Post hoc analyses revealed that the mean concreteness rating for neutral cue phrases $(M=5.36)$ was significantly higher than that for negative $(M=4.10)$ and positive $(M=3.93)$ cue phrases $(p s<.001)$, which did not differ from one another $(p=.18)$. The mean imagery rating for neutral $(M=5.36)$ cue phrases was also significantly higher than that for positive $(M=4.34)$ cue phrases $(p<.001)$, which was significantly higher than that for negative $(M=4.06)$ cue phrases $(p=.02)$. Thus, negative cue phrases were rated overall as being lower on imagery and concreteness than neutral phrases.

Autobiographical memory questionnaire. In Session 1, participants recorded details of their memories using a prestructured two-page questionnaire. The first page required participants to write a description of the content of the memory and to indicate whether the memory was triggered by something (either in one's thoughts or in the environment) or whether there was no trigger. If participants 
indicated that the memory was triggered, then they wrote a description of what the trigger was in their own words. Finally, they rated on a 5-point scale $(1=$ not at all concentrating; $5=$ fully concentrating) how much they were concentrating on the vigilance task when the memory came to mind. The second page of the questionnaire requested further details and evaluations of memory characteristics, such as the participants' ages in the memory, how often the memory had been thought of/rehearsed before ( 1 = never; 2 = sometimes; $3=$ often; $4=$ quite often; and $5=$ very often $)$, how unusual or common the remembered event was $(1=$ very common; $5=$ very unusual $)$, what the emotional valence of the memory was $(1=$ very negative; $3=$ neutral; 5 = very positive), and whether the remembered event was general or specific.

An identical, two-page questionnaire was used in Session 2 to record voluntary memories, with the exception that questions concerning the trigger of the memory and levels of concentration were omitted.

\section{Procedure}

Participants were tested individually. In Session 1, participants were told that they were taking part in a study examining concentration and that they would be required to carry out a vigilance task in which they had to detect a randomly presented target stimulus (consisting of patterns of vertical lines) from a large number of nontarget stimuli (consisting of patterns of horizontal lines). Each time a target stimulus was detected, participants had to respond by saying "yes" out loud (and the experimenter recorded their responses). Additionally, participants were informed that they would also see words in the center of the pattern, but they were instructed to ignore these. It was explained that the condition they were taking part in was looking at how people could keep their concentration on the patterns and that in another condition, participants would have to concentrate on the words and ignore the lines. At this point, participants were given a short, 1-min practice trial that contained three target stimuli.

Once the experimenter was assured that the participant understood the requirements of the vigilance task, it was explained that because the task was quite monotonous, they could possibly find themselves thinking about other things, which was quite normal. Thoughts unrelated to the vigilance task could concern many things - for example, a current project, future goals, daydreams, or memories from one's past - and it was explained that we were also interested in some of these unrelated thoughts. In particular, participants were informed that some unrelated thoughts could be past memories that spontaneously "pop" to mind, and the nature of involuntary autobiographical memory was explained. It was also pointed out that memories could be of specific or general events, from one's recent or remote past, and so forth.

Participants were instructed that their main task was to continue to respond by saying "yes" out loud each time they saw the target vertical lines, but that if an involuntary autobiographical memory came to mind, then they should click the mouse, which would stop the vigilance task and record their memory. Once the experimenter was assured that the instructions were understood, the participants started the task. If the participants clicked the mouse to indicate that a memory had come to mind, then this cleared the current trial/stimuli from the screen and presented a feedback screen with the message "Record your memory and then click the mouse to return to the vigilance task" for an unlimited time interval. Participants were handed a questionnaire and they completed the first page; the experimenter explained each question in detail for the first memory reported by each participant. After completing the first page of the questionnaire, participants clicked the mouse again to return to the vigilance task, repeating this procedure for each involuntary memory that came to mind. At the end of the task, the experimenter explained each question on the second page of the questionnaire, and the participants then completed one for each memory they had recorded during the vigilance task.

Session 2 took place approximately 1 week after Session 1, and it was undertaken in the same location and at approximately the same time. Participants were told that the computer screen would look very similar to that shown in the first session, because they would be shown several cards with words at the center of a pattern of horizontal lines. However, this time, they would be required to deliberately retrieve a past memory associated with the words presented on the screen. It was explained that each word phrase would remain on the screen for $1 \mathrm{~min}$ and that they should try to recall a past memory as quickly as possible. As soon as the memory was retrieved, they had to click the mouse, which would clear the current phrase from the screen and present a feedback screen with the message "Record your memory and then click the mouse to return to the task" for an unlimited time interval. During this time, the first page of the questionnaire would be completed. This procedure would then be repeated for each of the cue phrases presented. If participants were unable to recall a past memory within $1 \mathrm{~min}$, then the computer would automatically move on to the next word. It was also reiterated that past memories could be general or specific in nature and very recent or remote. Once all of the cue phrases had been presented, the participant completed page 2 of the questionnaire for each memory they had recorded.

\section{Data Analysis}

For Study 1 and Study 2, the data were treated in the same way. Before conducting any analyses, all involuntary and voluntary memories were read through to check that they were autobiographical in nature. Coding was carried out by two independent raters with high interrater reliability (ranging from $\kappa=.64, S E=.05$, to $\kappa=.84$, $S E=.06)$. Memories that were discarded were clearly not autobiographical in nature and were examples of future intentions (i.e., prospective memory), semantic "mind-pops" (see Kvavilashvili \& Mandler, 2004, for a detailed definition), tip-of-the-tongue phenomena, or current thoughts/daydreams.

For those involuntary memories that were reported by participants as being triggered by cues presented on the computer, it was possible to calculate retrieval times. When participants had an involuntary memory, they clicked the mouse, and the computer recorded the reaction time (RT). Retrieval times were calculated by counting from the present (clicked on) trial, back to the trial that presented the word that was reported by the participant to have triggered the involuntary memory. For example, if a participant clicked on Trial 23 and the RT for that trial was $0.84 \mathrm{sec}$, and the word that triggered the memory was two trials back, then $3.00 \mathrm{sec}$ would be added (i.e., $1.50 \mathrm{sec} /$ trial), to make a retrieval time of $3.84 \mathrm{sec}$.

Because participants provided multiple observations (i.e., memories) that could not truly be treated as independent, the majority of analysis involved using aggregated data (cf. Berntsen \& Hall, 2004). Thus, for each participant, we calculated means (e.g., mean retrieval time, rehearsal rating, etc.) that were then entered into the ANOVA. When the data were dichotomous (i.e., specific vs. general), mean proportions of specific memories were calculated for each participant. The frequencies of memories, instead of means, are reported only occasionally for descriptive purposes.

\section{Results}

\section{Descriptive Information}

All participants completed the vigilance task successfully, with an average of $14.38(S D=.37)$ targets detected (out of 15). Most importantly, only 4 out of 37 participants did not report any involuntary memories throughout the session. These participants were not invited back to take part in Session 2 on voluntary autobiographical memory.

After discarding 18 nonautobiographical memories (14 involuntary and 4 voluntary), the total number of involuntary memories recalled by the remaining 33 participants was 238 , with a mean of $7.21(S D=5.05$, range $=1-24)$ per participant. The total number of voluntary memories recalled in Session 2 was 602, with a mean of $18.24(S D=$ 5.22 , range $=5-24$ ). 
Out of 238 involuntary memories, the majority (i.e., $91 \%$ ) were reported to have identifiable triggers. Out of 217 memories with reported triggers, 201 (93\%) were triggered by phrases presented on the computer screen, with only 2 memories ( $1 \%$ ) being triggered by other environmental cues and $14(6 \%)$ being triggered by internal thoughts. It is also interesting that participants reported idiosyncratic sets of cue phrases. There were only 22 cues that were reported by more than 1 participant, with the majority $(n=14)$ being reported by 2 participants and the remaining 8 cues being reported by $3(n=4), 4(n=3)$, and $5(n=1)$ participants.

\section{Comparing Involuntary and Voluntary Memories}

Retrieval times. The retrieval times of those involuntary memories that were reported to have been triggered by a cue phrase in Session 1 were calculated individually for each memory, as was described at the end of the Method section. For voluntary memories in Session 2, the retrieval times were simply RTs from the moment participants saw the cue phrase on the screen until they pressed a button to report a memory. The mean retrieval time for involuntary memories was $5.06 \mathrm{sec}(S D=3.86)$, and it was significantly faster than the mean retrieval time of $9.74 \sec (S D=5.44)$ for voluntary memories $[F(1,31)=$ $\left.13.85, p=.001 ; \eta^{2}=.31\right]$.

Characteristics of memories. The overall mean ratings for all recorded memory characteristics (see Table 1) were entered into several repeated measures ANOVAs, with type of memory (involuntary vs. voluntary) as an independent variable. The mean proportion of specific involuntary memories $(M=.80)$ was significantly higher than that of voluntary autobiographical memories $(M=$ .69) $\left[F(1,32)=11.61, p=.002 ; \eta^{2}=.30\right]$. There were no differences between involuntary and voluntary memories in any other memory characteristics (largest $F=2.71$ ).

The relationship between memories and cues. First, we examined the number of memories as a function of cue valence and memory type. The mean number of involuntary and voluntary memories that were triggered by negative, positive, and neutral cues were calculated (see the top panel of Table 2) and entered into a 2 (memory type: involuntary, voluntary) $\times 3$ (cue valence: negative, neutral, positive) repeated measures ANOVA. Unsurprisingly, this analysis revealed a main effect of memory type $\left[F(1,31)=106.33, p<.001 ; \eta^{2}=.77\right]$; participants recalled more voluntary memories in Session 2 than they did involuntary memories in Session 1. The main effect of cue valence was not significant $(F<1)$. However, there was an interaction between memory type and cue valence $\left[F(1,30)=6.05, p=.006 ; \eta^{2}=.29\right]$. Followup tests showed that the number of voluntary memories retrieved to negative, neutral, and positive cues in Session 2 did not differ (smallest $p=.10$ ). However, the mean number of involuntary memories triggered by negative cues $(M=2.75, S D=1.97)$ was reliably higher than those triggered by positive $(M=1.94, S D=2.06)$ and neutral $(M=1.53, S D=1.63)$ cues $(p=.04$ and $p=.002$, respectively), which did not differ from each other $(p=.27)$.
In order to examine the correspondence between the valence of cue phrases and the participants' own ratings of the emotional valence of memories, overall mean emotional valence ratings were calculated as a function of cuetype (negative, neutral, positive) and were entered into a 2 (memory type: involuntary, voluntary) $\times 3$ (cue valence: negative, neutral, positive) repeated measures ANOVA (see Table 3 for means). There was a significant main effect of cue valence $\left[F(2,26)=32.46, p<.001 ; \eta^{2}=.71\right]$. Planned comparisons revealed that memories retrieved to positive cues were rated significantly higher $(M=3.97$, $S D=1.00)$ than memories retrieved to neutral cues $(M=$ $3.74, S D=0.73)(p=.02)$, which in turn were rated as more positive than those retrieved to negative cue phrases $(M=2.29, S D=1.00)(p=.02)$. The main effect of memory type and the memory type $\times$ cue valence interaction were not significant $\left(F_{\mathbf{s}}<1\right)$.

\section{Discussion}

Several novel findings emerged from Study 1. First, the results showed that involuntary memories were retrieved within $5 \mathrm{sec}$ and almost twice as fast as voluntary memories. In fact, their retrieval times were probably shorter,

Table 1

Overall Mean Proportions and Standard Deviations of Specific Memories, Ratings of Memory Characteristics, and Age of Memories As a Function of Memory Type in Study 1

\begin{tabular}{|c|c|c|c|c|}
\hline & \multicolumn{4}{|c|}{ Memory Type } \\
\hline & \multicolumn{2}{|c|}{ Involuntary } & \multicolumn{2}{|c|}{ Voluntary } \\
\hline & $M$ & $S D$ & $M$ & $S D$ \\
\hline Specificity $^{\mathrm{a}}$ & 0.80 & 0.15 & 0.69 & 0.16 \\
\hline Unusualness $^{\mathrm{b}}$ & 3.68 & 0.74 & 3.48 & 0.51 \\
\hline Rehearsal $^{\mathrm{b}}$ & 2.40 & 0.64 & 2.30 & 0.58 \\
\hline Emotion $^{\mathrm{b}}$ & 2.95 & 0.80 & 3.16 & 2.32 \\
\hline Age of memory (in years) ${ }^{c}$ & 4.85 & 4.15 & 4.25 & 3.13 \\
\hline
\end{tabular}

${ }^{a}$ Memories were rated as specific or general. Means represent mean proportions of specific memories averaged across participants. ${ }^{b}$ Ratings were made on 5 -point scales. For unusualness, scale points were $1=$ very common, $5=$ very unusual. For rehearsal, scale points were $1=$ never, 2 = sometimes, $3=$ often, $4=$ quite often, and $5=$ very often. For emotional valence, scale points were $1=$ very negative, $3=$ neutral, and $5=$ very positive. ${ }^{\mathrm{c}}$ For each memory, age was calculated by subtracting age in memory from current chronological age.

Table 2

Mean Number of Memories and Standard Deviations As a Function of Memory Type and Cue Valence in Study 1 and Study 2

\begin{tabular}{|c|c|c|c|c|c|c|}
\hline \multirow[b]{3}{*}{ Memory Type } & \multicolumn{6}{|c|}{ Cue Valence } \\
\hline & \multicolumn{2}{|c|}{ Negative } & \multicolumn{2}{|c|}{ Neutral } & \multicolumn{2}{|c|}{ Positive } \\
\hline & $M$ & $S D$ & $M$ & $S D$ & $M$ & $S D$ \\
\hline \multicolumn{7}{|c|}{ Study 1} \\
\hline Involuntary & 2.75 & 1.97 & 1.53 & 1.63 & 1.93 & 2.06 \\
\hline Voluntary & 5.94 & 1.61 & 6.31 & 1.90 & 6.22 & 2.25 \\
\hline \multicolumn{7}{|c|}{ Study 2} \\
\hline Involuntary & 2.41 & 1.87 & 1.18 & 1.27 & 1.44 & 1.45 \\
\hline Voluntary & 8.13 & 1.99 & 8.33 & 2.12 & 8.23 & 2.02 \\
\hline
\end{tabular}

Note - The larger number of voluntary memories in Study 2 is due to a larger number of cue words presented. 
Table 3

Mean Emotional Valence Ratings and Standard Deviations As a Function of Memory Type and Cue Valence in Study 1 and Study 2

\begin{tabular}{lccccc}
\hline & \multicolumn{4}{c}{ Type of Memory } \\
\cline { 2 - 3 } \cline { 5 - 5 } Cue Valence & \multicolumn{3}{c}{ Involuntary } & & \multicolumn{2}{c}{ Voluntary } \\
\cline { 2 - 3 } \cline { 5 - 6 } & $M$ & $S D$ & & $S D$ \\
\hline Negative & $\mathbf{2 . 2 9}$ & 1.00 & $\mathbf{2 . 1 2}$ & 0.63 \\
Neutral & $\mathbf{3 . 7 4}$ & 0.73 & $\mathbf{3 . 1 5}$ & 0.68 \\
Positive & $\mathbf{3 . 9 7}$ & 1.00 & $\mathbf{4 . 0 6}$ & 0.60 \\
& \multicolumn{5}{c}{ Study 2} \\
Negative & $\mathbf{2 . 6 7}$ & 0.92 & $\mathbf{2 . 5 3}$ & 0.56 \\
Neutral & $\mathbf{3 . 6 0}$ & 1.01 & $\mathbf{3 . 2 4}$ & 0.48 \\
Positive & $\mathbf{3 . 9 2}$ & 1.15 & $\mathbf{3 . 9 7}$ & 0.52 \\
\hline
\end{tabular}

Note-Ratings were made on a 5 -point scale $(1=$ very negative; $3=$ neutral; 5 = very positive).

since it could take participants 1 or $2 \mathrm{sec}$ (if not longer) to realize that what was passing through their minds was a memory from their past. This would reduce retrieval times to $2-3 \mathrm{sec}$ and would thus be similar to "directly retrieved" memories reported by Haque and Conway (2001). It is important, however, that involuntary memories were not of very recent events, as was suggested by Conway (2005). On average, they were between 4 and 5 years old and did not differ from voluntary memories.

On the other hand, interesting differences between the two forms of memory emerged for the hitherto unexplored variable - the emotional valence of cues. Thus, unlike voluntary memories, involuntary memories were more likely to be triggered by negative cues than by either positive or neutral cues. This finding cannot entirely be accounted for by lower ratings of concreteness and imagery obtained for negative cue phrases (see the Method section). If anything, research on voluntary autobiographical memories suggests that high-imagery concrete words are more effective cues than are more abstract words (e.g., Williams, Healy, \& Ellis, 1999). It is possible that this increased propensity of negative cues to elicit involuntary memories serves an adaptive function to warn or remind the person of similar events experienced in the past (cf. Schank, 1999).

Furthermore, results also showed that there was a high correspondence between the emotional valence of the cues and the participants' ratings of emotional valence of their involuntary and voluntary memories. Given that emotional valence of involuntary memories also affects current mood in a congruent way (see Berntsen, 1996), negative involuntary memories can therefore have a negative effect on a participant's current mood (Schlagman et al., 2007). This finding may have important practical implications for a variety of clinical conditions in which patients may be inadvertently exposed to negative cues and therefore would be vulnerable to experiencing specific negative involuntary autobiographical memories. Clearly, this is an interesting avenue for future research.

A final methodological point relates to the imagery and concreteness of cue phrases. Although analysis presented in the Method section showed that there were no differences between the cue phrases presented in the involuntary and voluntary sessions, there is a possibility that out of the 800 cue phrases presented in the involuntary session, participants recalled memories in response to those cues that had higher overall ratings of imagery and concreteness than the cue phrases used in the voluntary session. In order to examine this possibility, for each participant, we calculated the mean concreteness and imagery levels of those cue phrases that elicited/prompted their involuntary and voluntary memories, using the available ratings of these words from a previous sample of independent raters (see the Method section). The results of a one-way withinsubjects ANOVA showed that the participants' involuntary and voluntary cue phrases did not differ in imagery $(M=$ 4.67, $S D=0.45$, and $M=4.73, S D=0.08$, respectively) or concreteness $(M=4.62, S D=0.09$, and $M=4.69$, $S D=0.58$, respectively) $(F S<1)$.

\section{STUDY 2}

Although the results of Study 1 are theoretically important and may also have practical implications for some clinical populations, they should be treated with caution for several reasons. First, it is necessary to show that the findings are replicable across studies using different samples. The second important question that needs to be answered concerns the ecological validity of involuntary memories elicited in the laboratory: Are they representative of everyday involuntary memories that have been sampled in previous diary studies? (see Kvavilashvili \& Ellis, 2004). In order to address these questions, participants in Study 2 completed two laboratory sessions (like they did in Study 1); however, in addition, they kept a diary of their everyday involuntary memories during the week in between the two sessions. It was expected that although involuntary memories elicited in the laboratory would not be different from involuntary memories recorded in the diary, they would both differ from voluntarily retrieved autobiographical memories in specificity; that is, they would be more specific than voluntary memories.

An additional aim of Study 2 was to examine the possibility that participants in Study 1 tried to deliberately recall memories in the involuntary session to please the experimenter. In order to address the question about demand characteristics, participants in Study 2 completed a measure of social desirability (Crowne \& Marlowe, 1964). If involuntary memories are indeed deliberately recalled to please the researcher, then there should be a positive correlation between participants' social desirability scores and the number of reported involuntary memories.

Finally, several minor changes were made to the autobiographical memory questionnaire used in Study 1. First, a new question about the vividness of memory image was added to the questionnaire (see Berntsen \& Hall, 2004). second, the scale points for the rehearsal question were made more specific by asking participants how many times they had thought of the memory before (never, once or twice, a few times, several times, and many times) instead of how frequently they had thought of the memory (never, sometimes, often, quite often, and very often). The most important change concerned the question about the 
emotional valence of the memory (i.e., "What is the emotional valence of your memory?"). Since some participants in Study 1 needed clarification of the meaning of the term emotional valence, the wording of this question was changed to "How pleasant or unpleasant is your memory?" In addition, in Study 1, it was not quite clear whether participants rated the emotional valence of their memories per se, the original event when they first experienced it, or a mixture of both. Previous research has shown that retrospective evaluations of negative emotions become less intense over time (Walker, Vogl, \& Thompson, 1997) and that - at least in older adults - ratings of autobiographical memories become more positive over time (Field, 1981; Kennedy, Mather, \& Carstensen, 2004; Levine \& Bluck, 1997; Schlagman, Schulz, \& Kvavilashvili, 2006). To address this issue, an additional question requesting ratings of the pleasantness of the original event when it first occurred was included; that is, we distinguished between the pleasantness of the memory and of the original event. Doing so allowed us to examine whether the fading effect previously obtained on voluntary memories would also be present in involuntary memories.

\section{Method}

\section{Participants}

Forty-four psychology undergraduates (19 males and 25 females) with a mean age of 21.02 years $(S D=2.41$, range $=18-28)$ took part in the study in return for course credit. English was the first language of all participants.

\section{Materials and Procedure}

The materials and procedure used in Study 2 for Session 1 and Session 2 were very similar to those used in Study 1, except for the amendments and additions detailed below.

Session 1: Involuntary recall. The vigilance task that we used was a shortened version of that used in Study 1. The number of trials was reduced to 600 (11 target stimuli and 589 nontarget stimuli), which shortened the length of the SuperLab program to $15 \mathrm{~min}$. This reduction was made to take into account the cognitive slowing of older adults (ages 70-80) who also took part in the study. However, in this article, only the results of the young participants are reported.

Diaries. At the end of Session 1, all participants were provided with a diary (in the form of a notebook) containing 30 questionnaires1 to be completed for each involuntary autobiographical memory they experienced. They were given detailed verbal and written instructions of how to complete the diary. The nature of involuntary autobiographical memories was explained again, and it was reiterated that involuntary memories might be general or specific, recent or remote.

Participants carried the diaries with them at all convenient times for a period of 7 days, and they completed a questionnaire immediately (or as soon as possible) after the occurrence of an involuntary autobiographical memory. If participants were unable to complete the questionnaire immediately but later felt that they had forgotten key characteristics, then a space was provided for them to record this in the form of a check mark. There were no restrictions on how many memories were recorded each day.

Session 2: Voluntary recall. Participants were presented with 30 cue phrases ( 10 negative, 10 neutral, and 10 positive). The procedure for the voluntary autobiographical memory task was the same as that in Study 1. However, at the end of the session, participants completed the Marlowe-Crowne scale (Crowne \& Marlowe, 1964), which is a 33 -item measure of social desirability with a true-false response format. Typical items include I'm always willing to admit it when I make a mistake or I have never intensely disliked someone.
Scores can range from 0 to 33, with higher scores indicating greater social desirability.

Autobiographical memory questionnaire. The questionnaire used to record autobiographical memories was similar to the questionnaire used in Study 1, except for the following additions/ changes. On page 1 , an additional question required participants to rate the vividness of the memory on a 7 -point scale $(1=$ very vague, almost no image at all; 7 = very vivid, almost like normal vision). On page 2, an additional question concerned the emotional valence of the original event (How pleasant or unpleasant was the remembered event at the time you experienced it? 1 = very unpleasant $3=$ neutral; $5=$ very pleasant $)$. Finally, the wording was changed for the question concerning the emotional valence of the memory (How pleasant or unpleasant was your memory? 1 = very unpleasant $; 3=$ neutral $; 5=$ very pleasant $)$ and for the scale points of the question concerning prior rehearsal $(1=$ never; $2=$ once or twice; $3=$ afew times; $4=$ several times $; 5=$ many times $)$.

\section{Results \\ Number of Involuntary and Voluntary Autobiographical Memories}

In Session 1, all participants completed the vigilance task successfully, with an average of $10.70(S D=0.59)$ targets being detected out of a possible 11. Out of 44 participants, only 3 did not report experiencing any involuntary memories during Session 1. Having discarded 71 nonautobiographical memories (15 laboratory involuntary, 29 diary involuntary, and 27 voluntary), the remaining 41 participants reported a total of 251 involuntary memories, with a mean of $6.12(S D=3.81$, range $=1-18)$ per participant in Session 1. A total of 441 involuntary memories were recorded in full in the diaries over 7 days, with a mean of $10.05(S D=5.46$, range $=1-25)$ memories. In addition, a total of 308 involuntary memories were recorded in the diaries in the form of a check mark, with a mean of $7.16(S D=10.91$, range $=0-44)$. Finally, a total of 1,063 voluntary autobiographical memories were recalled during Session 2, with a mean of $24.16(S D=$ 6.10 , range $=8-30$ ) per participant.

The number of memories - regardless of type - was not related to participants' social desirability scores [laboratory memories, $r(43)=.02, p=.91$; diary memories, $r(43)=-.09, p=.58$; voluntary memories, $r(43)=.08$, $p=.64]$.

\section{Comparing the Retrieval Context of Laboratory and Diary Involuntary Memories}

Triggers. The majority of both laboratory (87\%) and diary $(77 \%)$ involuntary memories were reported to have been triggered as opposed to not triggered. However, the percentage of external triggers was higher for laboratory than for diary involuntary memories (79\% vs. 53\%), whereas this pattern was reversed for internal triggers $(8 \%$ vs. $24 \%$ ) and for no triggers ( $13 \%$ vs. $23 \%)$, where percentages were higher for diary memories $\left[\chi^{2}(2)=49.91\right.$, $p<.001]$. As in Study 1, out of the 217 laboratory involuntary memories that were triggered, 191 memories $(88 \%)$ were triggered by phrases presented on the computer screen, with only 7 memories (3\%) and 19 memories $(9 \%)$ triggered by other environmental cues and by internal thoughts, respectively. 
Concentration. The mean concentration rating for laboratory involuntary memories was $3.57(S D=.78$, range $=1-5$ ), and for diary involuntary memories, it was $3.03(S D=0.74$, range $=1-5)$. Thus, on average, participants reported medium levels of concentration. However, the results of the one-way repeated measures ANOVA showed that the mean concentration rating for laboratory memories was reliably higher than it was for diary memories $\left[F(1,39)=20.99, p<.001 ; \eta^{2}=.35\right]$.

\section{Comparing the Characteristics of Involuntary and Voluntary Memories}

Overall mean ratings of memory characteristics were entered into several one-way repeated measures ANOVAs, with type of memory (laboratory, diary, voluntary) as the independent variable (see Table 4 for means). There were no differences between laboratory, diary, and voluntary memories in vividness, unusualness, or age of memory (all $F_{S}<1.51$ ). However, there was a significant main effect of specificity $\left[F(2,80)=11.11, p<.001 ; \eta^{2}=.22\right]$ and of rehearsal $\left[F(2,80)=9.92, p<.001 ; \eta^{2}=.20\right]$. With regard to specificity, planned comparisons revealed that there was no difference between the proportions of specific laboratory and diary involuntary memories ( $p=$ .72). However, there was a significantly higher proportion of specific laboratory and diary involuntary memories than there was of voluntary memories $(p s<.001)$. Post hoc analysis of rehearsal ratings revealed that the mean rehearsal ratings for both laboratory and diary involuntary memories were higher than those of voluntary memories $(p<.001$ and $p=.01$, respectively). Although the mean rehearsal ratings of involuntary laboratory memories $(M=2.93)$ were somewhat higher than those for diary memories $(M=2.67)$, the difference was not statistically significant at the .05 level $(p=.06)$.

In order to examine the pleasantness of memories and of original events, overall means (also presented in Table 4) were entered into a 3 (memory type: laboratory, diary, voluntary) $\times 2$ (time: memory now, original event) repeated measures ANOVA. The analysis revealed a main effect of time $\left[F(1,40)=9.59, p=.004 ; \eta^{2}=.20\right]$, with pleasantness ratings of "now" $(M=3.30)$ being higher than pleasantness ratings of "then" $(M=3.12)$. The main effect of memory type was not significant $(F<1)$. There was, however, a significant memory type $\times$ time interaction $[F(1,80)=6.37$, $\left.p=.003 ; \eta^{2}=.14\right]$. Follow-up tests showed that there was a reliable difference between pleasantness ratings of memory now and of original event for laboratory involuntary memories $(p<.001)$; for diary involuntary memories, this difference was marginally significant $(p=.06)$. However, for voluntary memories, this difference was not significant $(p=.45)$. Thus, involuntary laboratory and diary memories were rated as more pleasant over time, but for voluntary memories, there was no difference between pleasantness ratings of the original event and the memory.

\section{Comparing Laboratory Involuntary and Voluntary Memories}

Retrieval times. Like in Study 1, the mean retrieval time of involuntary memories was $4.61 \mathrm{sec}(S D=3.81)$,
Table 4

Overall Mean Proportions and Standard Deviations of Specific Memories, Ratings of Memory Characteristics, and Age of Memories As a Function of Memory Type in Study 2

\begin{tabular}{|c|c|c|c|c|c|c|}
\hline & \multicolumn{6}{|c|}{ Type of Memory } \\
\hline & \multicolumn{2}{|c|}{ Laboratory } & \multicolumn{2}{|c|}{ Diary } & \multicolumn{2}{|c|}{ Voluntary } \\
\hline & $M$ & $S D$ & $M$ & $S D$ & $M$ & $S D$ \\
\hline Specificity $^{\mathrm{a}}$ & 0.79 & 0.17 & 0.80 & 0.15 & 0.67 & 0.10 \\
\hline Vividness ${ }^{b}$ & 4.95 & 1.07 & 4.86 & 0.85 & 5.11 & 0.78 \\
\hline Unusualness $^{\mathrm{c}}$ & 3.58 & 0.89 & 3.45 & 0.68 & 3.44 & 0.47 \\
\hline Rehearsal $^{\mathrm{c}}$ & 2.93 & 0.72 & 2.67 & 0.70 & 2.40 & 0.58 \\
\hline Memory pleasantness ${ }^{\mathrm{c}}$ & 3.40 & 0.68 & 3.29 & 0.70 & 3.21 & 0.57 \\
\hline Event pleasantness ${ }^{\mathrm{c}}$ & 3.04 & 0.90 & 3.19 & 0.81 & 3.15 & 0.28 \\
\hline Age of memory (in years) ${ }^{\mathrm{d}}$ & 3.85 & 3.32 & 3.67 & 2.69 & 4.07 & 1.75 \\
\hline
\end{tabular}

${ }^{\mathrm{a}}$ Memories were rated as specific or general. Means represent mean proportions of specific memories averaged across participants. ${ }^{\mathrm{b}}$ Ratings were made on a 7 -point scale $(1=$ very vague, almost no image at all; $7=$ very vivid, almost like normal vision). ${ }^{\mathrm{c}}$ Ratings were made on 5 -point scales. For unusualness, scale points were $1=$ very common, $5=$ very unusual. For rehearsal, scale points were $1=$ never, $2=$ once or twice, 3 = a few times, $4=$ several times, and $5=$ many times. For memory and event pleasantness, scale points were $1=$ very unpleasant, $3=$ neutral, and $5=$ very pleasant. ${ }^{\mathrm{d}}$ For each memory, age was calculated by subtracting age in memory from current chronological age.

which was significantly faster than the mean retrieval time of voluntary autobiographical memories $-10.13 \mathrm{sec}$ $(S D=4.26)\left[F(1,37)=41.08, p<.0001 ; \eta^{2}=.53\right]$.

The relationship between memories and cues. The mean number of involuntary and voluntary memories that were triggered/prompted by negative, positive, and neutral cues was calculated (see the bottom section of Table 2) and entered into a 2 (memory type: involuntary, voluntary) $\times 3$ (cue valence: negative, neutral, positive) repeated measures ANOVA. Like in Study 1, there was a significant interaction between memory type and cue valence $\left[F(2,76)=5.60, p=.005 ; \eta^{2}=.13\right]$. Post hoc comparisons showed that the mean number of voluntary memories retrieved to negative, neutral, and positive cues in Session 2 did not differ (smallest $p=.44$ ). However, the mean number of involuntary memories triggered by negative cues $(M=2.41)$ was reliably higher than the mean number of memories triggered by both positive cues $(M=1.18)$ and neutral cues $(M=1.44)(p=.01$ and $p<.001$, respectively), whereas the difference between the mean number of memories triggered by positive and neutral cues was not significant $(p=.40)$.

Finally, the relationship between cue valence and memory valence was examined. Overall mean pleasantness ratings for the memories were calculated as a function of cue type (negative, neutral, and positive) and were entered into a 2 (memory type: involuntary, voluntary) $\times 3$ (cue valence: negative, neutral, positive) repeated measures ANOVA (for means, see the bottom section of Table 3). Like in Study 1, there was a main effect of cue valence $\left[F(2,24)=19.29, p<.001 ; \eta^{2}=.62\right]$ (the lower degrees of freedom reflect the smaller number of participants included in this analysis, because not all participants reported memories to all three cue-valence categories). Planned comparisons showed that memories retrieved to positive cues were rated significantly higher $(M=3.94)$ than memories retrieved to neutral cues $(M=3.42)(p=$ 
.03), which, in turn, were rated as more positive than those retrieved to negative cue phrases $(M=2.60)(p=.003)$. The main effect of memory type and the memory type $x$ cue valence interaction were not significant $\left(F_{\mathrm{s}}<1\right)$.

\section{Discussion}

The results of Study 2 both replicated and extended the findings of Study 1 in several important ways. First, almost all of the comparisons between laboratory involuntary and voluntary memories resulted in findings that were identical to those in Study 1. Thus, involuntary laboratory memories were reported to be more specific than voluntarily retrieved memories, and their retrieval times were again reliably faster than those of voluntary memories. Additionally, there was a high correspondence between the emotional valence of the cues and the participants' ratings of how pleasant their memories were for both laboratory involuntary memories and voluntary memories. Finally, like in Study 1, voluntary memories were equally likely to be retrieved to negative, neutral, and positive cues; however, a larger number of involuntary memories were triggered by negative cues than by positive or neutral cues.

The inclusion of an additional diary study of involuntary autobiographical memories in Study 2 allowed us to test the ecological validity of the laboratory involuntary memories by comparing them with the involuntary diary memories recorded by the same participants. The findings showed that there were no differences between them in terms of memory characteristics. However, both laboratory and diary involuntary memories were found to differ from voluntarily retrieved memories on several important variables. First, involuntary laboratory and involuntary diary memories were more specific than voluntary memories. second, the results showed that the so-called fading effect (i.e., memories being rated as more positive now than the original event) was present in both laboratory and diary involuntary memories, but not in the voluntarily retrieved memories in Session 2. The fading effect has previously been found in voluntary autobiographical memories (see e.g., Walker et al., 1997). However, in the present study, the pleasantness ratings of voluntary memories and original event did not differ. The discrepant findings for voluntary memories could be partly due to some methodological differences between the studies. Indeed, in most of the previous studies, participants had to rate the pleasantness of the same memories at several retention intervals, with ratings of these memories becoming more positive over time (Walker et al., 1997), whereas in the present study, the ratings of memories and the original event were obtained at the same point in time.

Finally, both laboratory and diary involuntary memories were more rehearsed than voluntary memories. Although this finding further emphasizes similarities between laboratory and diary involuntary memories, it contradicts the results of Study 1; no differences were found between involuntary and voluntary memories in rehearsal ratings. This lack of replication could be due to different scales being used for rehearsal ratings. In Study 2, the scale was more refined and included options of once or twice and a few times as opposed to sometimes and often used in Study 1. It should be pointed out, however, that the mean rehearsal ratings for all three types of memories are fairly low and below point 3, which represents a few times on the scale.

An additional and methodologically important finding was that the number of involuntary memories experienced during the laboratory session was not related to scores of social desirability, indicating that participants were not reporting involuntary memories because of demand characteristics.

\section{GENERAL DISCUSSION}

The major aim of this article was to systematically examine the similarities and differences between involuntary and voluntary autobiographical memories by comparing their memory characteristics and, importantly, their retrieval processes. An additional aim was to test the validity of the new method by comparing involuntary memories elicited in the laboratory with everyday involuntary memories recorded in diaries. In this section, we will first summarize the most important findings from the two studies and then discuss their theoretical implications for current research of autobiographical memory. This will be followed by a brief discussion of some methodological issues - including criticisms of our method - as well as potential avenues for future research.

The first set of findings concerns the direct comparisons between involuntary and voluntary memories. The results showed that involuntary and voluntary memories did not differ in several memory characteristics, such as vividness, unusualness, pleasantness, and age of the memory. However, major differences between the two types of memories emerged for several other variables. For example, involuntary memories were significantly more likely to be of specific events than were voluntary memories, which replicates the findings of previous diary studies that also compared the specificity of involuntary and voluntary autobiographical memories (see Berntsen, 1998; Berntsen \& Hall, 2004). Most importantly, involuntary memories were retrieved within 4-5 sec and almost twice as fast as voluntary memories. Thus, specific involuntary memories were coming to mind relatively quickly and without any deliberate effort, whereas voluntary memories, retrieved in Session 2, provide further support to the general view that the retrieval of autobiographical memories is slow and effortful, taking time to construct specific events (see Rubin, 1998; Rubin \& Schulkind, 1997a, 1997b).

An additional difference between involuntary and voluntary memories concerns the emotional valence of cues. Thus, voluntary memories were as likely to be retrieved to negative, positive, and neutral cues, whereas involuntary memories were more likely to be triggered by negative cues. This finding would have been impossible to obtain without a laboratory method, and it may have interesting theoretical implications. Thus, if negative stimuli in the environment are more likely to trigger past experiences, this may act as either a warning sign of what happened previously or as a reassurance that the previous outcome was not negative. Therefore, it can be 
argued that in everyday life, these involuntary memories may serve some adaptive function by protecting individuals from potentially harmful or unpleasant events/experiences (cf. Schlagman et al., 2006). This idea is somewhat in line with the "warning signal" hypothesis in relation to the intrusive memories experienced by individuals with post-traumatic stress disorder (PTSD) (see, e.g., Ehlers et al., 2002). Intrusive memories in PTSD are often of events that occurred just prior to the traumatic experience; for example, a patient who had experienced a head-on car crash at night kept seeing headlights coming toward her (Ehlers et al., 2002; Ehlers, Michael, Chen, Payne, \& Shan, 2006). Therefore, it is assumed that one mechanism behind such memories might be to warn the individual of immediate danger. In patients with PTSD, such memories are usually always triggered, but not necessarily by negative stimuli. For example, the intrusive memory described above was often triggered by any round patches of light on a dark surface. Thus, intrusive memories might be an extreme manifestation of a possible normal function of involuntary memories.

The second set of findings concerns the comparisons between laboratory and diary involuntary memories. The results showed that they did not differ in any memory characteristics (e.g., specificity, emotional valence, rehearsal ratings, age of memory). In addition, when compared with voluntary memories, they showed similar patterns of differences in specificity, rehearsal, and the fading effect. Taken together, these findings indicate that the involuntary memories elicited in the laboratory are representative of involuntary memories experienced in everyday life. Despite the similarities there were some minor differences in number of reported memories and external triggers. Thus, on average, six memories were reported per participant in the laboratory session, whereas only two to three memories were recorded per day in diaries (for similar results, see Berntsen, 1996, 1998; Kvavilashvili \& Mandler, 2004). The number of reported external triggers was also higher in the laboratory than in the diary. However, these differences are hardly surprising, given that in the laboratory, participants were engaged in a relatively undemanding vigilance task and were exposed to hundreds of cues within a short space of time. In everyday life, people are often engaged in more demanding activities and thus may be less likely to notice potential cues.

\section{Theoretical Implications}

The results of this article have important theoretical implications for current research on autobiographical memory. For example, they provide initial evidence that the socalled "directly retrieved" memories, which are observed occasionally in voluntary memory experiments, and the involuntary memories, which are spontaneously recalled when one is not in the "retrieval" mode, may be quite similar and possibly based on the same retrieval mechanism. Although involuntary memories in the present study were recalled within 4-5 sec, these somewhat longer RTs were most likely due to the fact that participants needed a few seconds to realize that what they were thinking about was actually a memory (cf. Schooler, Reichle, \& Halpern,
2005). Therefore, the "true" retrieval time of involuntary memories may be approximately $2-3 \mathrm{sec}$, which is similar to that of directly retrieved memories in the voluntary memory experiments.

The important question that needs to be answered concerns the underlying mechanisms of these "directly retrieved" memories and involuntary memories. Are they mediated by reconstructive or reproductive retrieval processes? According to the most influential model of autobiographical memory, knowledge of one's past is distributed across a hierarchical system (Conway \& Pleydell-Pearce, 2000). Therefore, specific memories are not represented by a single memory trace; rather, "memories are transitory dynamic mental constructions" (p. 261) that are formed by combining information from various levels of the autobiographical memory knowledge base (see also Conway, 2005). Thus, voluntary retrieval of autobiographical memories follows the hierarchy top-down as lifetime period information enables access to general events, which in turn facilitates access to fragmentary sensory, perceptual, and affective information that represents the specific details of a past event.

In contrast, with respect to involuntary autobiographical memories, the prevailing theoretical assumption is that they are directly retrieved from a separate pool of memories. Berntsen (1998), for example, proposed a "double model" of autobiographical memory in which two different autobiographical knowledge systems exist. One system records and stores specific memories as single representations; the other contains more abstract information, such as lifetime periods. On occasion, these specific memories can be directly accessed in response to salient cues. Similarly, Conway (2005) suggested that involuntary memories are directly retrieved from a separate pool of recent memories that have not yet been consolidated into the long-term autobiographical memory system.

However, the results of the present study - concerning the retrieval times and characteristics of involuntary memories - do not seem to support the views of Berntsen (1998) and Conway (2005). Indeed, involuntary memories were not of very recent events, since their mean age was between 4 and 5 years in both studies (see Ball \& Little, 2006 , for similar results). In addition, approximately $20 \%$ of the involuntary memories concerned general as opposed to specific events, which goes against the idea that these memories are retrieved from a separate pool of most recent and specific memories.

The only way to explain these findings is to suggest that involuntary memories are retrieved from the same autobiographical memory knowledge base as voluntary memories and that their retrieval follows the same top-down (and probably reconstructive) process proposed for voluntary memories. However, the major difference between involuntary and voluntary autobiographical memories lies in the way in which the information in the autobiographical memory system is activated. The spreading of activation during voluntary retrieval is deliberately directed by the rememberer in a conscious effort to recall. In contrast, the spreading of activation during involuntary retrieval occurs automatically, without conscious awareness, and 
in response to some accidental stimuli in one's environment or thoughts. Additional support for this idea comes from the retrieval time data. Indeed, our results show that it takes, on average, between 4 and $5 \mathrm{sec}$ to report an involuntary autobiographical memory, with a "true" retrieval time possibly being 1 or $2 \mathrm{sec}$ shorter. If the spreading of activation in response to a cue within the system is fast and automatic, then it should be possible to reach the bottom of the hierarchy and construct a specific memory within this time period. Moreover, if this top-down spread of activation is, for some reason, terminated or interrupted before reaching lower levels of hierarchy, then this will result in the recall of general involuntary memories, as discussed previously.

A diary study of Kvavilashvili and Mandler (2004) on involuntary semantic memories or mind-pops (i.e., a sudden occurrence of contents of one's semantic knowledge, such as someone's name, a word, or a tune) has shown that encountered stimuli elicit fairly long-lasting activations in the network of one's semantic knowledge. For example, hearing the word "actor" may subconsciously activate the names of several actors so that later on, one of these names pops up unexpectedly. The results of the present study suggest that encountered stimuli might also elicit the spread of activations in the network of autobiographical knowledge as well. If such automatic spread of activation did not occur in response to the cue phrases encountered on the screen, then it would be impossible to explain the occurrence of involuntary memories in response to some of these stimuli. In fact, one could argue that in the voluntary memory experiments, participants start to deliberately search for a memory only when this fast and automatic spread of activation in response to a cue word does not result in a formation of a memory. When it does, the participant experiences the so-called "direct retrieval"; a memory seems to pop up even before the participant starts a deliberate search for the memory in response to a cue.

Therefore, an important theoretical question that remains to be answered is why certain stimuli in the environment elicit an involuntary memory and the others do not? In other words, what are the factors that determine whether a certain pattern of activation in the autobiographical knowledge base - instigated by the stimulireaches consciousness in the form of involuntary memory? One interesting possibility is that for a cue to trigger a particular memory, this memory or its fragments should have already been partially activated in the recent past (see Berntsen, 2007). It is interesting that initial support for this priming hypothesis was obtained by Mace (2005) in a study in which participants completed a diary of their involuntary autobiographical memories for a period of 2 weeks. At the end of the first week, participants were asked to voluntarily recall memories from a specific lifetime period (e.g., high school, or when they were between 13 and 16 years old) for $30 \mathrm{~min}$. After doing so, participants continued to record their involuntary memories in a diary for an additional week. The results showed that participants who had been primed for a particular lifetime period in the laboratory involuntarily recalled a larger pro- portion of memories from this period during the following week than did the controls. Importantly, these memories were not identical to memories deliberately recalled in the laboratory; they were of different events but from the same time period.

An alternative possibility is that for a cue to elicit an involuntary memory, there must be a perfect match between the content of the cue (e.g., hearing a song about red roses) and the central features of a particular memory (e.g., receiving a bunch of red roses on your first date) (see, e.g., Ball, Mace, \& Corona, 2007; Berntsen, 1998; Schlagman et al., 2007). This could explain the relatively small number of memories reported by participants in our studies even though they were exposed to hundreds of cues during the vigilance task. One logical implication of this position is that the same cue would elicit the same involuntary memory every time it is encountered (due to its perfect match with the central features of that particular memory). However, everyday observations suggest that this may not be the case. The most plausible assumption would be that the elicitation of a particular involuntary memory is due to some combination of these two factors. Clearly, this would be an interesting avenue for future research.

\section{Methodological Issues}

Apart from theoretical input, the results of the present article also have important methodological implications for research on involuntary memories. First, they show that involuntary memories can be elicited in the laboratory; the majority of participants did report one or more involuntary memories during the undemanding vigilance task. second, the majority of memories were triggered by irrelevant cue phrases presented on the screen, and this allowed us to reliably measure the retrieval times of involuntary memories and compare them with voluntary memories. Third, it was also possible to manipulate the emotional valence of cues and observe their effects on the number and the character of elicited memories.

Since developing the new method in 2003 (Kvavilashvili \& Schlagman, 2003), the importance of laboratorybased methods for studying involuntary autobiographical memory has been emphasized by several other researchers who have also started working on the design of such methods. For example, Mace (2006) had participants deliberately recall autobiographical memories in response to cue words and report any involuntary memories triggered by these voluntary memories (so-called "memory chaining"). The results showed that in $46 \%$ of cases, the recall of a voluntary memory was followed by the recall of an involuntary memory. On the other hand, Ball (2007) used a method in which participants had to generate continuous free associations to word cues for 20-30 sec per word while being tape-recorded. At the end of the session, participants listened to their taped free associations and indicated if and/or when they had experienced an involuntary memory. Involuntary autobiographical memories were reported during $86 \%$ of trials. Finally, Watkins et al. (2005) used a 30-min think-aloud task while simultaneously presenting a range of stimuli (music, pictures, and scents) to participants. Any thoughts considered to be 
autobiographical memories were noted by the experimenter and verified with the participant at the end of the session. If the participant agreed that it was a memory, then he or she had to indicate whether the memory had come to mind involuntarily or voluntarily.

The methods used by Ball (2007) and Watkins et al. (2005) are different from the method in the present article in that they both rely on participants' retrospective evaluations. On the other hand, like the present method, Mace (2006) attempted to catch involuntary autobiographical memories "online" as they occurred during the session, but could only sample involuntary memories that were triggered by the preceding voluntarily retrieved memories. However, involuntary memories triggered in this way occur fairly infrequently in everyday life. For example, in a diary study by Mace (2005), these memories constituted only $10 \%-12 \%$ of all recorded involuntary memories. Therefore, what makes the method developed in this article different is that it attempts to simulate the conditions in which involuntary autobiographical memories occur in everyday life. One major advantage of the method lies in its ability to allow researchers to measure the retrieval times of involuntary autobiographical memories. In contrast, an advantage of the methods used by Ball and by Watkins et al. is that they do not inform the participants of the nature of involuntary autobiographical memories in advance, which reduces or eliminates the possibility of demand characteristics in the experiment.

Clearly, by informing participants of the concept of involuntary memories in advance, there is the risk that they might assume that it is the main focus of the study and deliberately retrieve memories to please the experimenter. It is also possible that participants may have tried to recall memories in order to bring some variety into the (somewhat) monotonous vigilance task. However, in Study 2, demand characteristics were examined by taking measures of social desirability, but no relationship was found between social desirability scores and the number of memories reported. Most importantly, if participants were deliberately retrieving memories in the involuntary memory session, then it is unclear why they recalled more specific memories - and did so almost twice as fast-as they did in the voluntary session.

Another potential criticism of our method is that differences obtained between involuntary and voluntary memories were due to different demands of the two sessions; participants had to retrieve and record a larger number of voluntary memories than involuntary memories. In addition, participants always carried out the voluntary session last, which raises issues of potential order effects. Although these criticisms are valid, it is unlikely that they can entirely account for the obtained pattern of results. First, retrieval times and specificity of memories were examined for the first 10 voluntary memories retrieved, and the results remained the same. For example, in Study 2, the average retrieval time for the first 10 voluntary memories was $9.07 \mathrm{sec}(S D=4.79)$, and the mean proportion of specific memories was .62 $(S D=.17)$. second, the delay between the two sessions was 1 week, which makes it unlikely that involuntary memories retrieved in Ses- sion 1 affected voluntary memories in Session 2. Also, in a recent study, Ball (2007) counterbalanced the order in which participants carried out involuntary and voluntary memory tasks. Although Ball found differences between involuntary and voluntary memories in their specificity, importantly, he did not find any effect of task order.

Despite potential criticisms, it is clear that studying involuntary autobiographical memories under more controlled laboratory conditions is beneficial for future research. A range of issues that were either difficult or impossible to study with the diary method alone can begin to be examined. For example, the strength of the relation between elicitation triggers and involuntary memories could be examined by having participants carry out the same vigilance task some months after the first session. By providing the same set of cues, one could assess whether cues directly map to a memory (in which case, the same cue would always elicit the same memory) or whether other mechanisms, such as priming, are predominantly involved in the retrieval of involuntary autobiographical memories (see Mace, 2005).

Although diary studies of involuntary memory are important for a thorough understanding of the phenomenon, the present method - along with other laboratory-based methods that have been reported recently (Ball, 2007; Mace, 2006; Watkins et al., 2005)—will undoubtedly stimulate further research in this area (see also Ball, 2007). Therefore, with methodologies that can be easily manipulated, we may begin to systematically address questions pertaining to the nature, functions, and mechanisms of involuntary autobiographical memory.

\section{AUTHOR NOTE}

The research presented in this article was supported by a doctoral studentship awarded to S.S. from the Economic and Social Research Council, U.K. Portions of the research described in this article were presented at the Autobiographical Memory Meeting (Oxford University) in January 2005 and the 6th Biennial Meeting of the Society of Applied Research in Memory and Cognition (University of Wellington, New Zealand) in January 2005. We are grateful to David Rubin, Rachel Msetfi, and anonymous reviewers for their helpful comments and suggestions on earlier drafts of the manuscript. Correspondence concerning this article should be addressed to L. Kvavilashvili, School of Psychology, University of Hertfordshire, College Lane, Hatfield, Herts., AL10 9AB, England (e-mail: 1.kvavilashvili@herts.ac.uk).

\section{REFERENCES}

BALl, C. T. (2007). Can we elicit involuntary autobiographical memories in the laboratory? In J. H. Mace (Ed.), Involuntary memory (pp. 127-152). Malden, MA: Blackwell.

BALl, C. T., \& LitTle, J. C. (2006). A comparison of involuntary autobiographical memory retrievals. Applied Cognitive Psychology, 20, 1167-1179.

Ball, C. T., Mace, J. H., \& Corona, H. (2007). Cues to the gusts of memory. In J. H. Mace (Ed.), Involuntary memory (pp. 113-126). Malden, MA: Blackwell.

BARSALOU, L. W. (1988). The content and organization of autobiographical memories. In U. Neisser \& E. Winograd (Eds.), Remembering reconsidered: Ecological and traditional approaches to the study of memory (pp. 193-243). Cambridge: Cambridge University Press.

Baumeister, R. F., Bratslavsky, E., Finkenauer, C., \& Vohs, K. D. (2001). Bad is stronger than good. Review of General Psychology, 5, 323-370.

Berntsen, D. (1996). Involuntary autobiographical memories. Applied Cognitive Psychology, 10, 455-460. 
Berntsen, D. (1998). Voluntary and involuntary access to autobiographical memory. Memory, 6, 113-141.

BERNTSEN, D. (2007). Involuntary autobiographical memories: Speculations, findings, and an attempt to integrate them. In J. H. Mace (Ed.), Involuntary memory (pp. 20-49). Malden, MA: Blackwell.

Berntsen, D., \& HaLl, N. M. (2004). The episodic nature of involuntary autobiographical memories. Memory \& Cognition, 32, 789-803.

Berntsen, D., \& Rubin, D. C. (2002). Emotionally charged autobiographical memories across the lifespan: The recall of happy, sad, traumatic, and involuntary memories. Psychology \& Aging, 17, 636-652.

Conway, M. A. (2005). Memory and the self. Journal of Memory \& Language, 53, 597-628.

Conway, M. A., \& Bekerian, D. A. (1987). Organization in autobiographical memory. Memory \& Cognition, 15, 119-132.

Conway, M. A., \& Pleydell-Pearce, C. W. (2000). The construction of autobiographical memories in the self-memory system. Psychological Review, 107, 261-288.

Crowne, D. P., \& Marlowe, D. A. (1964). The approval motive: Studies in evaluative dependence. New York: Wiley.

Ehlers, A., Hackmann, A., Steil, R., Cohessy, S., Wenninger, K., \& WinTER, H. (2002). The nature of intrusive memories after trauma: The warning signal hypothesis. Behaviour Research \& Therapy, 40, 995-1002.

Ehlers, A., Michael, T., Chen, Y., Payne, E., \& Shan, S. (2006). Enhanced perceptual priming for neutral stimuli in a traumatic context: A pathway to intrusive memories? Memory, 14, 316-328.

FIELD, D. (1981). Retrospective reports by healthy intelligent older people of personal events of their adult lives. International Journal of Behavioral Development, 4, 77-97.

Giambra, L. M. (1989). Task-unrelated thought frequency as a function of age: A laboratory study. Psychology \& Aging, 4, 136-143.

Giambra, L. M. (1995). A laboratory method for investigating influences on switching attention to task unrelated imagery and thought. Consciousness \& Cognition, 4, 1-21.

Hall, N. M., \& Berntsen, D. (2008). The effect of emotional stress on involuntary and voluntary conscious memories. Memory, 16, 48-57.

Haque, S., \& Conway, M. A. (2001). Sampling the process of autobiographical memory construction. European Journal of Cognitive Psychology, 13, 529-547.

Kennedy, Q., Mather, M., \& Carstensen, L. L. (2004). The role of motivation in the age-related positivity effect in autobiographical memory. Psychological Science, 15, 208-214.

Kvavilashvili, L., \& Ellis, J. (2004). Ecological validity and twenty years of real-life/laboratory controversy in memory research: A critical and historical review. History \& Philosophy of Psychology, 6, 59-80.

KVaVilashvili, L., \& Mandler, G. (2004). Out of one's mind: A study of involuntary semantic memories. Cognitive Psychology, 48, 47-94.

Kvavilashvili, L., \& Schlagman, S. (2003, July). Eliciting involuntary autobiographical memories in the laboratory: Developing a new method of investigation. Paper presented at the 5th Biennial Meeting of the Society of Applied Research on Memory and Cognition (SARMAC), University of Aberdeen, Scotland.

LEVINE, L. J., \& BLUCK, S. (1997). Experienced and remembered emotional intensity in older adults. Psychology \& Aging, 12, 514-523.

Linton, M. (1986). Ways of searching and the contents of memory. In D. C. Rubin (Ed.), Autobiographical memory (pp. 50-66). Cambridge: Cambridge University Press.

MACE, J. H. (2004). Involuntary autobiographical memories are highly dependent on abstract cueing: The Proustian view is incorrect. $A p$ plied Cognitive Psychology, 18, 893-899.
MACE, J. H. (2005). Priming involuntary autobiographical memories. Memory, 31, 874-884.

MACE, J. H. (2006). Episodic remembering creates access to involuntary conscious memory: Demonstrating involuntary recall on a voluntary recall task. Memory, 14, 917-924.

Mace, J. H. (2007). Involuntary memory: Concept and theory. In J. H. Mace (Ed.), Involuntary memory (pp. 1-19). Malden, MA: Blackwell.

RuBIN, D. C. (1998). Beginnings of a theory of autobiographical remembering. In C. T. Thompson, D. J. Herrmann, D. Bruce, J. D. Read, D. G. Payne, \& M. Toglia (Eds.), Autobiographical memory: Theoretical and applied perspectives (pp. 47-67). Mahwah, NJ: Erlbaum.

Rubin, D. C. (2005). Autobiographical memory tasks in cognitive research. In A. Wenzel \& D. C. Rubin (Eds.), Cognitive methods and their application to clinical research (pp. 219-241). Washington, DC: American Psychological Association.

Rubin, D. C., \& Kozin, M. (1984). Vivid memories. Cognition, 16, 81-95.

Rubin, D. C., \& Schulkind, M. D. (1997a). The distribution of autobiographical memories across the lifespan. Memory \& Cognition, 25, 859-866.

Rubin, D. C., \& Schulkind, M. D. (1997b). Distribution of important and word-cued autobiographical memories in 20-, 35-, and 70-yearold adults. Psychology \& Aging, 12, 524-535.

Schank, R. C. (1999). Dynamic memory revisited. Cambridge: Cambridge University Press.

Schlagman, S., Kvavilashvili, L., \& Schulz, J. (2007). Effects of age on involuntary autobiographical memories. In J. H. Mace (Ed.), Involuntary memory (pp. 87-112). Malden, MA: Blackwell.

Schlagman, S., Schulz, J., \& Kvavilashvili, L. (2006). A content analysis of involuntary autobiographical memories: Examining the positivity effect in old age. Memory, 17, 161-175.

Schooler, J. W., Reichle, E. D., \& HALPERn, D. V. (2005). Zoning-out during reading: Evidence for dissociations between experience and meta-consciousness. In D. T. Levin (Ed.), Thinking and seeing: Visual metacognition in adults and children (pp. 204-226). Cambridge, MA: MIT Press.

SINGER, J. L. (1993). Experimental studies of ongoing conscious experience. In G. R. Bock \& J. Marsh (Eds.), Experimental and theoretical studies of consciousness (pp. 100-122). Chichester, U.K.: Wiley.

Smallwood, J. M., \& Schooler, J. (2006). The restless mind. Psychological Bulletin, 132, 946-958.

Walker, W. R., Vogl, R. J., \& Thompson, C. P. (1997). Autobiographical memory: Unpleasantness fades faster than pleasantness over time. Applied Cognitive Psychology, 11, 399-413.

Watkins, P. C., Grimm, D. L., Whitney, A., \& Brown, A. (2005). Unintentional memory bias in depression. In A. V. Clark (Ed.), Mood state and health (pp. 59-86). Hauppage, NY: Nova Science.

Williams, J. M. G., \& Dritschel, B. H. (1992). Categoric and extended autobiographical memories. In M. A. Conway, D. C. Rubin, H. Spinnler, \& W. A. Wagenaar (Eds.), Theoretical perspectives on autobiographical memory (pp. 391-410). Dordecht: Kluwer.

Williams, J. M. G., Healy, H. G., \& Ellis, N. C. (1999). The effect of imageability and predictability of cues in autobiographical memory. Quarterly Journal of Experimental Psychology, 52A, 555-579.

(Manuscript received February 21, 2007; revision accepted for publication February 4, 2008.) 